\title{
A full polar cap cascade model: pulsar $\gamma$-ray and X-ray luminosities
}

\author{
Bing Zhang and Alice K. Harding \\ NASA Goddard Space Flight Center, Greenbelt, MD 20771, USA
}

\begin{abstract}
We propose a full polar cap cascade model which includes the curvature and inverse Compton emission of the primary particles, and both synchrotron radiation and inverse Compton of the higher generation pairs. Such a full cascade model can reproduce both the $L_{\gamma} \propto$ $\left(L_{\mathrm{sd}}\right)^{1 / 2}$ and the $L_{x} \sim 10^{-3} L_{\mathrm{sd}}$ dependences observed from the known spin-powered pulsars.
\end{abstract}

The $8 \gamma$-ray and $35 \mathrm{X}$-ray spin-powered pulsars show the empirical laws of $L_{\gamma} \propto$ $\left(L_{\mathrm{sd}}\right)^{1 / 2} \propto B / P^{2}$ (Thompson et al. 1997), and $L_{x} \sim 10^{-3} L_{\mathrm{sd}} \propto B^{2} / P^{4}$ (Becker \& Trümper 1997), where $L_{\text {sd }}$ is the spin-down luminosity of the pulsar. Such features could be reproduced within the framework of a full polar cap cascade model. The basic picture of such a model is: primary particles accelerated from the inner gap emit primary $\gamma$-rays via curvature radiation (CR) or inverse Compton scattering (ICS) with the soft thermal photons near the surface, these $\gamma$-rays will pair produce in strong magnetic fields. The secondary pairs have nonzero pitch angles with respect to the field lines. The perpendicular energy of the pairs will be converted to radiation via synchrotron radiation (SR), and the parallel energy of the pairs will also be converted to radiation via ICS. These ICS branches of the pairs, which were neglected in the canonical polar cap cascade models, are important in two respects. Firstly, since usually the scattering occurs in the resonant regime, the energy conversion rate to radiation is almost $100 \%$. As a result, almost all the primary particles' kinetic energy is converted to high energy radiation, and hence, the high energy radiation luminosity (mainly the $\gamma$-ray luminosity) is identical to the polar cap particle luminosity, which is directly available from a certain acceleration model. Secondly, the soft tail of these ICS branches can contribute some non-thermal emission components in the X-ray band, which all the SR branches could not reach. This gives a natural interpretation to the observed non-thermal X-ray emission from some pulsars.

An analytical description of such a full cascade model was carried out (Zhang \& Harding 2000) within the framework of the Harding \& Muslimov (1998) acceleration model, in which the space-charge-limited flow model was improved by incorporating the frame-dragging $E_{\|}$, upper and lower pair formation front and both CR and ICS of the primary electrons. The predicted total high energy luminosity (mainly $\gamma$-ray luminosity) of this model is $L_{\gamma} \simeq 9.4 \times$ $10^{31} \mathrm{erg} \cdot \mathrm{s}^{-1} B_{p, 12}^{6 / 7} P^{-13 / 7} R_{E, 6}^{4 / 7}(\cos \alpha)^{8 / 7}$ for young pulsars in which $E_{\|}$in the accelerators are not saturated, or $L_{\gamma} \simeq 1.6 \times 10^{31} \mathrm{erg} \cdot \mathrm{s}^{-1} B_{p, 12} P^{-9 / 4} R_{E, 6}^{2}(\cos \alpha)^{5 / 4}$ for older and millisecond pulsars with saturated accelerators, where $P, B_{p}, R_{E}$ and $\alpha$ are pulsar period, surface magnetic field, effective stellar radius, and in- 


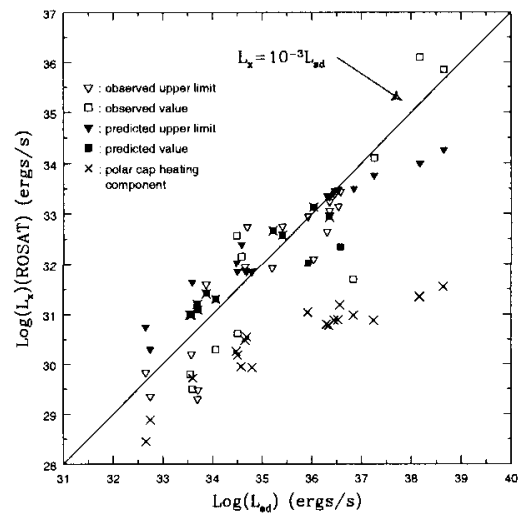

Figure 1. ROSAT-band X-ray luminosities: observation versus theory.

clination angle, respectively. Self-consistent polar cap heating in such a model produces a hot polar cap thermal component with temperature $T_{\mathrm{pc}, \max } \simeq 2.2 \times$ $10^{6} \mathrm{~K} B_{p, 12}^{1 / 14} P^{-1 / 14} R_{E, 6}^{-2 / 7}(\cos \alpha)^{5 / 28}$ or $T_{\mathrm{pc}, \max } \simeq 0.53 \times 10^{6} \mathrm{~K} B_{p, 12}^{1 / 4} P^{-9 / 16} r_{e, 6}^{-1 / 2} R_{E, 6}^{5 / 4}$ $(\cos \alpha)^{5 / 16}$ for unsaturated and saturated accelerators, respectively. This thermal component and another full surface thermal component due to neutron star cooling act as the soft photon sources of the various ICS processes. The basic ingredients of our analytic approach to describe the full cascade processes are, the energy portions of both the perpendicular (SR) branch and the parallel (ICS) branch; the recursion relations between different generations, i.e., the reduction factors of the typical photon energy between adjacent generations, and some non-integer generation order parameters, etc. With these ingredients, one can construct an analytic expression of the non-thermal X-ray luminosity within a certain band (Zhang \& Harding 2000). The sum of this non-thermal-component luminosity with other two thermal-component luminosities gives the prediction of our model. A comparison of the model predictions with the observations of the ROSAT-band luminosities of the known rotation-powered pulsars are shown in Fig.1. One can see that the $L_{x} \sim 10^{-3} L_{\text {sd }}$ feature is well-reproduced. One conclusion is that in millisecond pulsars, the thermal component due to polar cap heating dominates the ROSAT-band luminosity, if there is no strong multipole magnetic fields near the star surface.

\section{References}

Becker, W., \& Trümper, J. 1997, A\&A, 326, 682

Harding, A.K., \& Muslimov, A.G. 1998, ApJ, 508, 328

Thompson, D.J., Harding, A.K., Hermsen, W., \& Ulmer, M.P. 1997, in C.D. Dermer, M.S. Strickman, \& J.D. Kurfess (eds.), Proc. Fourth Compton Symposium, AIP Conf. Proc. 410, 39

Zhang, B., \& Harding, A.K. 2000, ApJ, 532, in press (astro-ph/9911028) 